\section{Análisis del sistema de alistamiento y priorización para trasplante hepático de adultos en Chile}

\author{
RODRIGO WOLFF ${ }^{1}$, LUIS ANTONIO DÍAZ ${ }^{1}$, BLANCA NORERO ${ }^{1}$, \\ ÁLVARO URZÚA ${ }^{2}$, GABRIEL MEZZANO ${ }^{3}$, ROBERTO HUMERES ${ }^{4}$, \\ FRANCO INNOCENTI ${ }^{5}$, LORENA CASTRO ${ }^{6}$, CLAUDIA PAVEZ $^{7}$, \\ CARLOS DEROSAS ${ }^{8}$, SUSANA ELGUETA $^{9}$
}

Comité asesor para alistamiento y priorización para trasplante hepático en Chile

\section{Analysis of the organ allocation system for liver transplantation in Chile}

Background: In Chile, organ allocation for liver transplantation (LT) in adults is prioritized according to the MELD-Na score. Exceptions such as Hepatocellular Carcinoma (HCC) and other non-HCC exceptions receive a score called Operational MELD score. Aim: To evaluate the effectiveness of the MELD-Na score and the operational MELD score as a prioritization system for LT in Chile. Material and Methods: Retrospective analysis of the waiting list (WL) of adult candidates ( $\geq 15$ years) for elective LT in Chile from 2011 to 2017. The probability of leaving the WL, defined by death or contraindication for $L T$ was compared in three groups: 1) Cirrhotic patients prioritized according to their real MELD-Na score (CPM), 2) HCC and 3) other non-HCC exceptions. Results: We analyzed 730 candidates for LT, with a median age of 57 years, 431 (56\%) were men. In the study period, $352 \mathrm{LT}$ were performed (48\%). The annual exit rate was significantly higher in the CPM group (45.5\%) compared to HCC (33.1\%) and non-HCC (29.3\%), ( $p<0.001)$. Post LT survival was $86 \%$ at 1 year and $85 \%$ at 5 years, without significant differences between groups. In the CPM group, post-transplant survival was significantly lower $(p<0.05)$ in patients with $\mathrm{MELD}-\mathrm{Na} \geq 30$ at transplant ( $81 \%$ per year) compared to patients with patients with MELD-Na $<30$ (91\% per year). Conclusions: $M E L D-N a$ score can discriminate very well patients who have a higher risk of death in the short and medium term. However, the assignment of operational scores for situations of exception produces inequities in the allocation of organs for LT and must therefore be carefully adjusted.

(Rev Med Chile 2020; 148: 1541-1549)

Key words: Carcinoma, Hepatocellular; Waiting Lists; Liver Transplantation; Tissue and Organ Procurement.

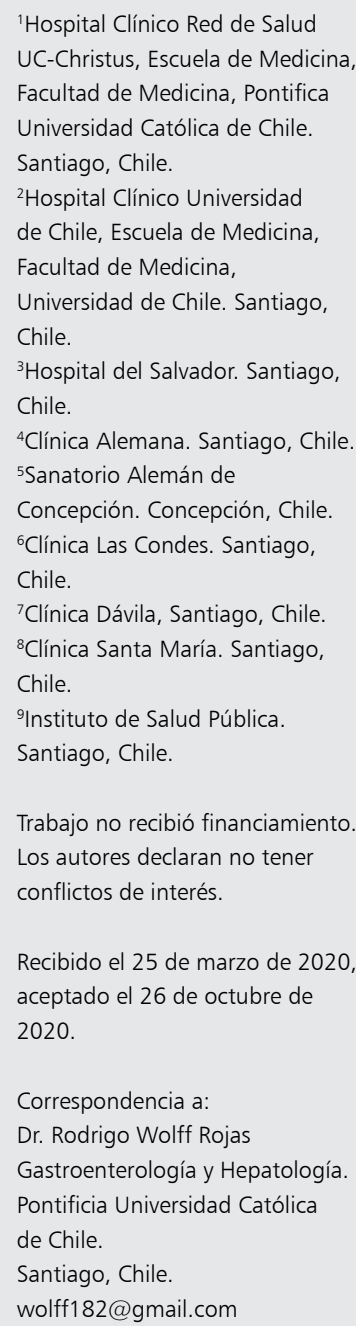

1 trasplante hepático es actualmente considerado una terapia estándar para el tratamiento de la enfermedad hepática terminal, ya que mejora la supervivencia y es costo-efectiva. En Chile se han desarrollado programas de trasplante hepático exitosos en varios centros, realizándose entre 70 y 100 trasplantes anuales, los que a la fecha suman más de 1.600 trasplantes hepáticos, con resultados de supervivencia comparables a los países desarrollados ${ }^{1,2}$.

La principal fuente de órganos para trasplante hepático en adultos en Chile son los donantes 
cadavéricos. Durante los últimos 10 años, las tasas de donación en Chile han oscilado entre 7 y 10 donantes por millón de habitantes (dpm), lo cual es muy bajo en comparación con Europa y Estados Unidos de Norteamérica (22 y $35 \mathrm{dpm}$, respectivamente) y otros países de nuestra región como Brasil, Argentina y Uruguay (17, 19 y 23 dpm, respectivamente $)^{3}$. Estas bajas tasas de donación, en parte, se explican por la negativa familiar $(50 \%)$, pero también por una deficiente red de detección de potenciales donantes, fallas en la asistencia del donante, problemas en la coordinación de equipos de procura, descartes de órganos por hígado graso y otras causas. Por otro lado, la lista de espera (LE) para trasplante hepático ha ido creciendo sostenidamente en los últimos años: en el año 2000 era de 30 pacientes, llegando a su punto más alto en 2008 , con 240 pacientes. Luego al implementar el sistema MELD (Model for End-stage Liver Disease) con criterio mínimo de alistamiento un puntaje mayor de 15, se disminuyó la LE a 130 pacientes, aproximadamente (Figura 1).

La escasez de órganos disponibles y la mayor demanda han determinado una elevada tasa de salida de la LE por muerte o progresión de la enfermedad más allá de lo recomendable para trasplante. Por otro lado, se requiere trasplantar pacientes más graves, lo que aumenta los costos, los días de hospitalización y potencialmente podría impactar en la supervivencia postrasplante ${ }^{4}$. Dada esta escasez, es necesario contar con un sistema de priorización que permita una distribución justa de los órganos disponibles y un manejo racional de la lista de espera.

En Chile, inicialmente la priorización de la LE para trasplante hepático se basó en el tiempo de espera, ocasionando que muchos tratantes alistasen pacientes precozmente, para asegurar la posibilidad de trasplante, mientras que otros pacientes con mayor necesidad fallecieran en la espera. En la búsqueda de un sistema más justo y equitativo, que priorizara a los enfermos con mayor probabilidad de muerte, desde octubre del 2011 se adoptó el sistema de puntuación MELD para la asignación de órgano para trasplante hepático y, desde diciembre de 2016, se modificó al sistema MELD-Na. El sistema de puntuación MELD es un modelo de predicción de supervivencia a corto y mediano plazo en la enfermedad hepática que utiliza 3 variables de laboratorio: creatininemia, bilirrubinemia y tiempo de protrombina, medido como INR (Internatinal Normalization Ratio $)^{5,6}$; y el MELD-Na agrega el sodio plasmático a la ecuación ${ }^{7,8}$. Si bien este modelo se desarrolló para predecir mortalidad frente al uso de TIPS (transjugular intra-hepatic porto-systemic shunt), ha sido ampliamente utilizado para priorizar a los enfermos en la LE de trasplante hepático.

Sin embargo, existen muchas condiciones, incluido el carcinoma hepatocelular (CHC), en las que el MELD o MELD-Na no reflejan la mortalidad o la probabilidad de salida de la LE. En

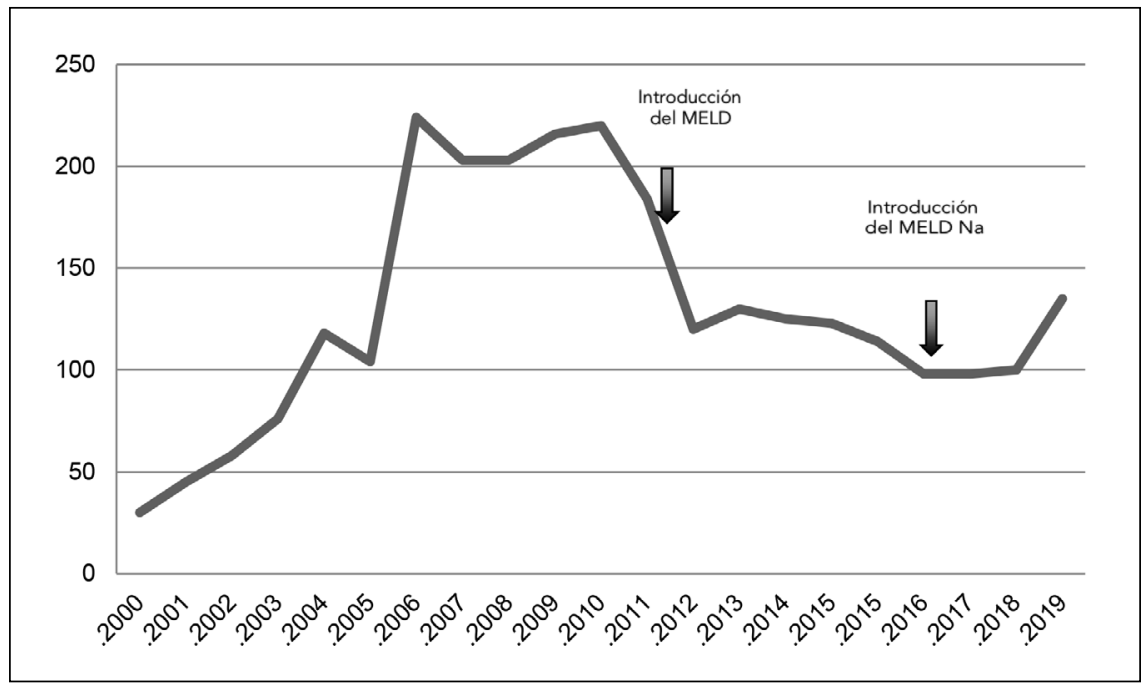

Figura 1. Evolución del número de pacientes en lista de espera de trasplante hepático. Introducción del MELD en octubre de 2011 y de MELD-Na en diciembre de 2016. 
estas situaciones se asigna un puntaje adicional de excepción, que intenta reflejar la mayor gravedad de la condición, llamado MELD operacional. La actual normativa para priorización en LE en Chile asigna 20 puntos de MELD operacional más un punto adicional cada 2 meses para los candidatos con $\mathrm{CHC}$ mayor a $2 \mathrm{~cm}$, dentro de criterios de Milán (con 1 lesión $\leq 50 \mathrm{~mm}$ o hasta 3 lesiones con la mayor $\leq 30 \mathrm{~mm})^{2,9}$. Otras condiciones de excepción como encefalopatía hepática refractaria recibe 15 puntos más un punto cada 3 meses; ascitis refractaria 15 puntos más un punto cada 3 meses; prurito refractario 15 puntos más un punto cada 3 meses; síndrome hepatopulmonar recibe 20 puntos más un punto cada 3 meses. La enfermedad hepática poliquística, que raramente produce disfunción hepática, pero puede disminuir la calidad de vida y luego mejorar con el trasplante hepático, recibe 15 puntos más un punto cada 3 meses.

Estos puntajes operacionales fueron asignados por el Comité asesor para alistamiento y priorización para trasplante hepático en Chile, en base a la mortalidad esperada a 3 meses y a la experiencia de los miembros del comité10-13.

El objetivo de nuestro estudio fue evaluar la efectividad de la puntuación MELD-Na y el puntaje de MELD operacional como sistema de priorización para trasplante hepático en Chile. Se comparó el abandono en la lista de espera entre los candidatos cirróticos priorizados según su MELD$\mathrm{Na}$ real (grupo CPM) y los candidatos priorizados por excepciones con puntaje MELD operacional, ya sea por $\mathrm{CHC} \mathrm{u}$ otras excepciones (no-CHC). Adicionalmente se evaluó la supervivencia postrasplante para los distintos grupos.

\section{Métodos}

Se realizó un análisis retrospectivo de la LE de candidatos adultos ( $\geq 15$ años) para trasplante hepático electivo con donante cadavérico en Chile, desde el 15 de octubre de 2011 al 31 de diciembre de 2017. Se excluyeron los candidatos con falla hepática fulminante, candidatos pediátricos $(<15$ años), retirados de LE por consumo activo de sustancias de abuso, contraindicación quirúrgica antes de la fecha de inicio del estudio y candidatos que perdieron sus seguimientos.

Todos los datos fueron procesados de manera anónima, utilizando la codificación cifrada del Instituto de Salud Pública. Este estudio fue aprobado por el Comité de Ética de la Pontificia Universidad Católica de Chile (ID 190424001).

El principal resultado fue la probabilidad de salida de LE definida por muerte, mala condición física que contraindique el trasplante o la progresión de la enfermedad en CHC (fuera de los criterios de Milán), para lo cual se compararon tres grupos: 1) Candidatos cirróticos priorizados según su MELD o MELD-Na real (grupo CPM); 2) Candidatos con carcinoma hepatocelular ( $\mathrm{CHC}) ; 3$ ) Candidatos con otras excepciones (no-CHC). En estas comparaciones, se utilizó el puntaje MELD-Na real en candidatos con cirrosis hepática sin excepciones y el puntaje MELD operacional en pacientes con $\mathrm{CHC}$ y otras excepciones no-CHC.

Además, se comparó la probabilidad de muerte en el grupo de cirróticos en LE de acuerdo al MELD-Na al momento del alistamiento. Para dicho análisis, solo se incluyó pacientes alistados sin excepciones.

Según tipo de distribución, los datos continuos se describieron utilizando la media y deviación estándar, o mediana y rangos intercuartílicos (RIQ). Los datos nominales se describieron utilizando porcentajes. Para el análisis de las proporciones se utilizó las pruebas $\chi^{2}$ o exacta de Fisher. Se analizó la supervivencia en LE y postrasplante hepático representadas en curvas de Kaplan-Meier con prueba log-rank. El análisis estadístico se realizó con programa IBM SPSS versión 22.0 (IBM Corp; Armonk, NY, USA).

\section{Resultados}

Desde octubre de 2011 a diciembre de 2017 se alistaron 773 candidatos adultos para trasplante hepático electivo, de los cuales se excluyeron 43 pacientes por no cumplir con criterios de inclusión. La mediana de la edad al alistamiento fue de 57 años (RIQ 49-62,7 años), 431 (55,8\%) eran hombres. Las principales etiologías fueron enfermedad por hígado graso no alcohólica (EHGNA) $(27,7 \%)$, enfermedad hepática alcohólica $(15,2 \%)$ y hepatitis autoinmune $(11,1 \%)$ (Tabla 1$)$. De los pacientes alistados, $313(42,9 \%)$ correspondieron al grupo CPM, 195 (26,7\%) CHC y $222(30,4 \%)$ no-CHC (Tabla 2). En el período de estudio se realizaron 352 trasplantes $(48,2 \%)$. La principales causas de excepción fueron encefalopatía recurrente, ascitis refractaria y prurito intratable (Tabla 3). 
Alistamiento y priorización para trasplante hepático en adultos en Chile - R. Wolff et al

Tabla 1. Principales etiologías de pacientes alistados para trasplante hepático por hepatopatías crónicas en adultos

\begin{tabular}{|lrl|}
\hline Causa & n & $\mathbf{( \% )}$ \\
\hline Esteatohepatitis no alcohólica & 202 & $(27,7)$ \\
\hline Alcohol & 111 & $(15,2)$ \\
Hepatitis autoinmune & 81 & $(11,1)$ \\
Hepatitis virales & 75 & $(10,3)$ \\
$\quad$ Virus hepatitis C & 72 & $(9,9)$ \\
Virus hepatitis B & 3 & $(0,4)$ \\
Enfermedades colestásicas & 57 & $(7,8)$ \\
Colangitis biliar primaria & 31 & $(4,2)$ \\
Síndrome de sobreposición & 16 & $(2,2)$ \\
Colangitis esclerosante primaria & 8 & $(1,1)$ \\
Cirrosis biliar secundaria & 2 & $(0,3)$ \\
Otros & 145 & $(19,9)$ \\
No precisado & 59 & $(8)$ \\
Total & 730 & $(100)$ \\
\hline
\end{tabular}

La mediana de MELD-Na real al alistamiento fue de 20 puntos para toda la cohorte. La mediana de MELD operacional al momento del trasplante fue de 28 puntos (RIQ 23-33) en el grupo CPM, 25 puntos (RIQ 21-28) en el grupo CHC y 25 puntos (RIQ 23-29) en el grupo no-CHC. El tiempo medio en lista de espera al trasplante fue de 198 días: 116 (RIQ 27-337) días en el grupo CPM, 263 (RIQ 154-457) días en el grupo CHC y 248 (RIQ 72-477) en el grupo no-CHC (Tabla 4).

La tasa de salida de LE al año, el principal resultado del estudio, fue significativamente mayor en el grupo CPM $(45,5 \%)$ en comparación con los candidatos con $\mathrm{CHC}(33,1 \%)$ y las excepciones no-CHC $(29,3 \%)$, respectivamente $(\mathrm{p}<0,001)$ (Tabla 5 y Figura 2).

En el grupo CPM, la probabilidad de mortalidad a 90 días tuvo una fuerte correlación con el MELD-Na al momento de ingreso a la lista

Tabla 2. Características sociodemográficas de los candidatos alistados para trasplante hepático por hepatopatías crónicas en adultos

\begin{tabular}{|lc|}
\hline Variables & Frecuencia (n = 730) (\%) \\
\hline Edad (años) $[$ IQ 25-75]* & $57[49-62,7]$ \\
\hline Género masculino & $55,8 \%$ \\
\hline Grupo sanguíneo & $417(57,1)$ \\
O & $246(33,7)$ \\
A & $48(6,6)$ \\
B & $19(2,6)$ \\
AB & \\
Causa de alistamiento & $313(42,9)$ \\
Cirróticos sin excepciones (PC) & $195(26,7)$ \\
Candidatos con hepatocarcinoma (CHC) & $233(30,4)$ \\
\hline Otras excepciones no hepatocarcinoma (No CHC) & \\
\hline
\end{tabular}

*Mediana [rango intercuartil 25-75].

Tabla 3. Causas de excepciones no CHC

\begin{tabular}{|lcccc|}
\hline Causa de excepción & $\mathbf{n}$ & $\mathbf{( \% )}$ & $\begin{array}{c}\text { Trasplantados } \\
\mathbf{n}(\%)\end{array}$ & $\begin{array}{c}\text { Espera promedio al } \\
\text { TH (días) }\end{array}$ \\
\hline Encefalopatía recurrente & 57 & $(26)$ & $26(45)$ & 427 \\
Ascitis refractaria & 50 & $(23)$ & $23(46)$ & 261 \\
\hline Prurito intratable & 42 & $(19)$ & $22(52)$ & 397 \\
\hline Enfermedad poliquistica & 16 & $(7)$ & $5(75)$ & 549 \\
\hline Colangitis recurrente & 11 & $(5)$ & $13(81)$ & 327 \\
Sd. hepatopulmonar & 16 & $(7)$ & $16(65)$ & 230 \\
\hline Otros & $30(14)$ & & & 358 \\
\hline
\end{tabular}


Tabla 4. Puntaje MELD al alistamiento y al trasplante según grupos: cirróticos sin excepciones (PC); candidatos con hepatocarcinoma (CHC); otras excepciones no hepatocarcinoma (No CHC)

\begin{tabular}{|lccrc|}
\hline & Grupo PC & Grupo CHC & Grupo No CHC & Valor p \\
\hline MELD operacional al alistamiento* & $21[17-26]$ & $20[20-20]$ & $17[15-22]$ & $<0,001$ \\
\hline MELD real al TH* & $28[22-33]$ & $12[9-15]$ & $22[15-32]$ & $<0,001$ \\
\hline MELD operacional al TH* & $28[23-33]$ & $25[21-28]$ & $25[23-29]$ & 0,012 \\
\hline
\end{tabular}

*Mediana [rango intercuartil 25-75]. TH: trasplante hepático.

Tabla 5. Probabilidad de salida de lista de espera y tiempos promedio en lista según grupos: cirróticos priorizados por MELD (CPM); candidatos con carcinoma hepatocelular (CHC); otras excepciones no carcinoma hepatocelular (No CHC)

\begin{tabular}{|lcccc|}
\hline Variables & $\begin{array}{c}\text { Grupo CPM } \\
\text { (N = 313) }\end{array}$ & $\begin{array}{c}\text { Grupo CHC } \\
\mathbf{( N = 1 9 5 )}\end{array}$ & $\begin{array}{c}\text { Grupo No CHC } \\
\text { (N = 222) }\end{array}$ & Valor p \\
\hline Salida de lista (\%) & & & & 0,005 \\
$\quad 1$ año & $44 \%$ & $33,4 \%$ & 30,8 & \\
3 años & $71,5 \%$ & $66 \%$ & $79,4 \%$ & \\
5 años & $81 \%$ & $69,7 \%$ & $89,7 \%$ & $<0,001$ \\
\hline Tiempo de espera en lista (días) & $116[27-337]$ & $263[154-457]$ & $248,5[72-477]$ & $<$ \\
\hline
\end{tabular}

*Mediana [rango intercuartil 25-75].

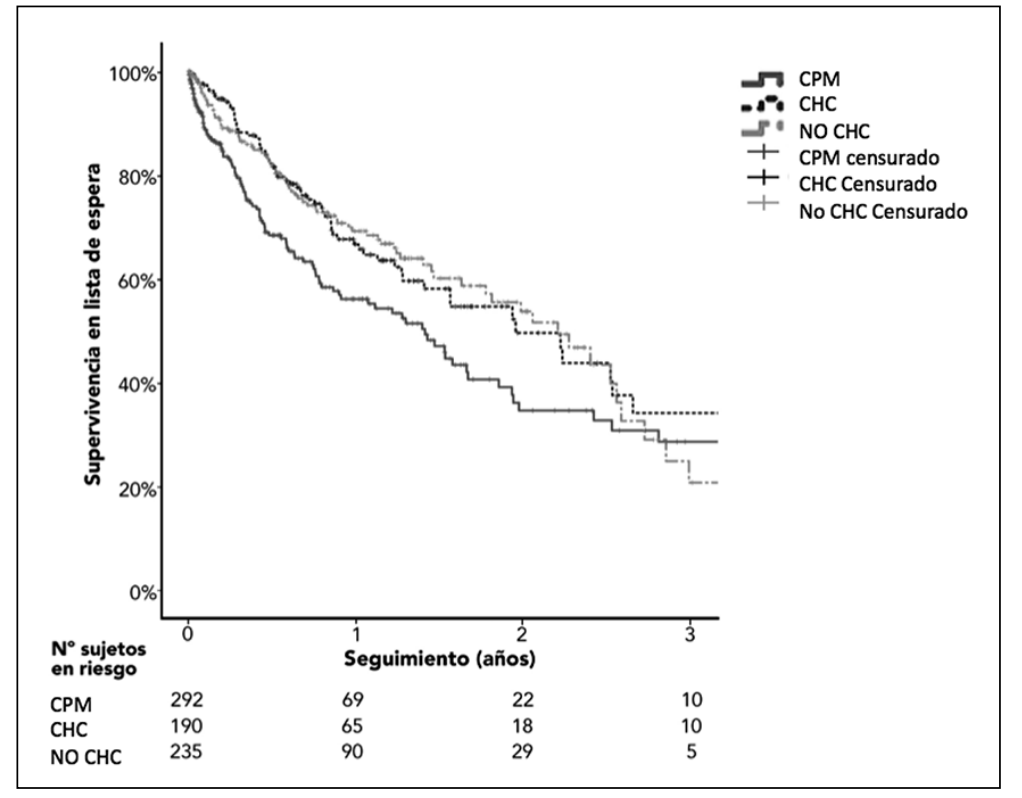

Figura 2. Supervivencia en lista de espera en pacientes cirróticos (CPM), con carcinoma hepatocelular (CHC) y excepciones no carcinoma hepatocelular (No $\mathrm{CHC}$ ). de espera con un R de 0,79 (IC 95\% 0,73-0,86) (Figuras 3 y 4 ).

La supervivencia posterior al TH fue de $86,1 \%$ a 1 año y $84,6 \%$ a 5 años, sin diferencias significativas $(p>0,05)$ entre los tres grupos (Figura 5).
Dentro del grupo CPM, la supervivencia postrasplante fue significativamente menor $(\mathrm{p}<0,05)$ en pacientes con MELD-Na a $\geq 30$ al momento del trasplante ( $81 \%$ al año), comparado con pacientes con MELD < 30 (91\% al año) (Figura 6). 


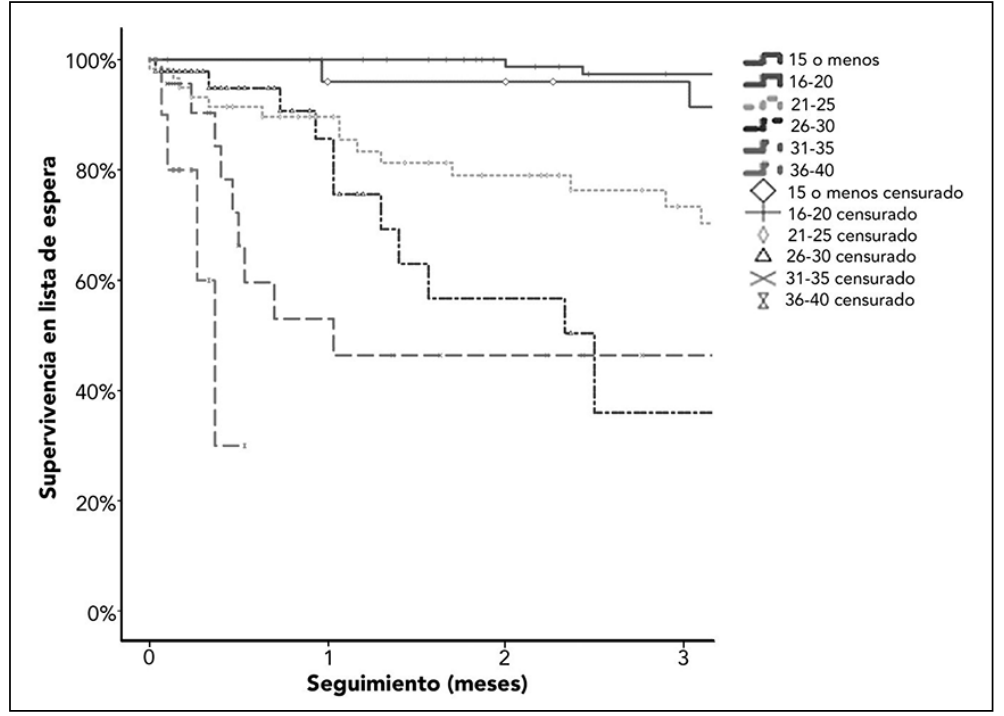

Figura 3. Supervivencia en lista de espera en pacientes cirróticos (grupo CPM) según MELD agrupado al momento del alistamiento.

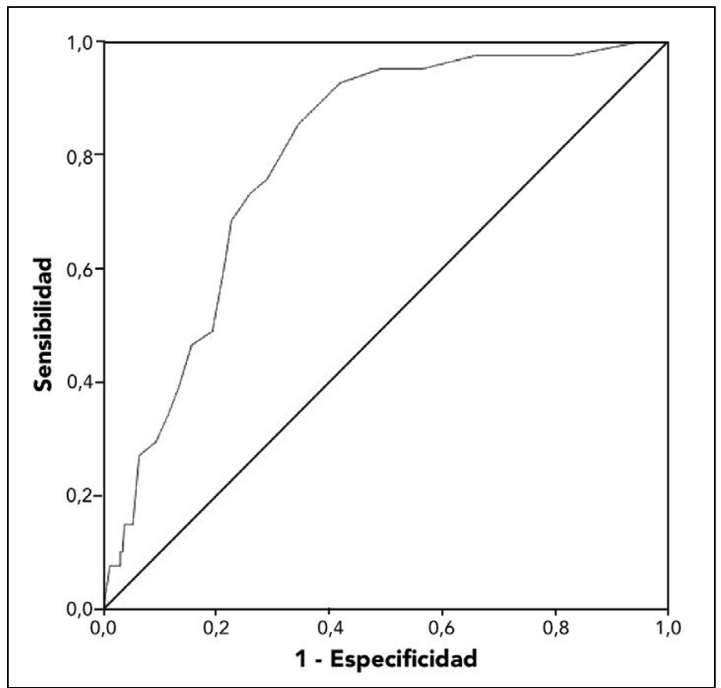

Figura 4. Curva ROC para predicción de mortalidad a 90 días del MELD-Na en el grupo de pacientes cirróticos priorizados según su MELD-Na real (CPM).

\section{Discusión}

El sistema MELD y su variante MELD-Na son ampliamente utilizados en el mundo para el alistamiento y priorización para trasplante hepático, ya que son sencillos y de fácil acceso en centros de mediana complejidad, permitiendo una distribu- ción más justa de los escasos órganos disponibles a quienes más lo necesitan. Fue implementado en Estados Unidos de Norteamérica en 2002, extendiendo su uso posteriormente a Europa, Latinoamérica y algunos países de Asia ${ }^{6,14-16}$.

Se han descrito algunas desventajas a este método, por ejemplo, la cuantificación de creatinina plasmática puede no ser un buen predictor de función renal en pacientes con cirrosis, ya que estos suelen tener un grado importante de desnutrición y sarcopenia, lo que puede subestimar la disfunción renal ${ }^{17,18}$. Por similar razón, las mujeres, quienes por tener una menor masa muscular, pueden verse perjudicadas en las listas de priorización ${ }^{19}$. Los métodos de medición de electrolitos plasmáticos pueden tener un margen de error no despreciable, especialmente cuando hay hiponatremia significativa, con lo que pequeños cambios pueden significar una variación importante en el MELD-Na final.

Sin embargo, a pesar de sus debilidades, es un método objetivo que permite identificar pacientes con mayor probabilidad de mortalidad a corto y mediano plazo. En el presente estudio se encontró una fuerte correlación entre la mortalidad a 90 días y el MELD-Na al momento de ingreso a la lista (R 0,79) (Figura 3), lo cual valida en nuestra población la utilidad del MELD como sistema de priorización de pacientes por gravedad y pronóstico. En un estudio previo, en una cohorte 

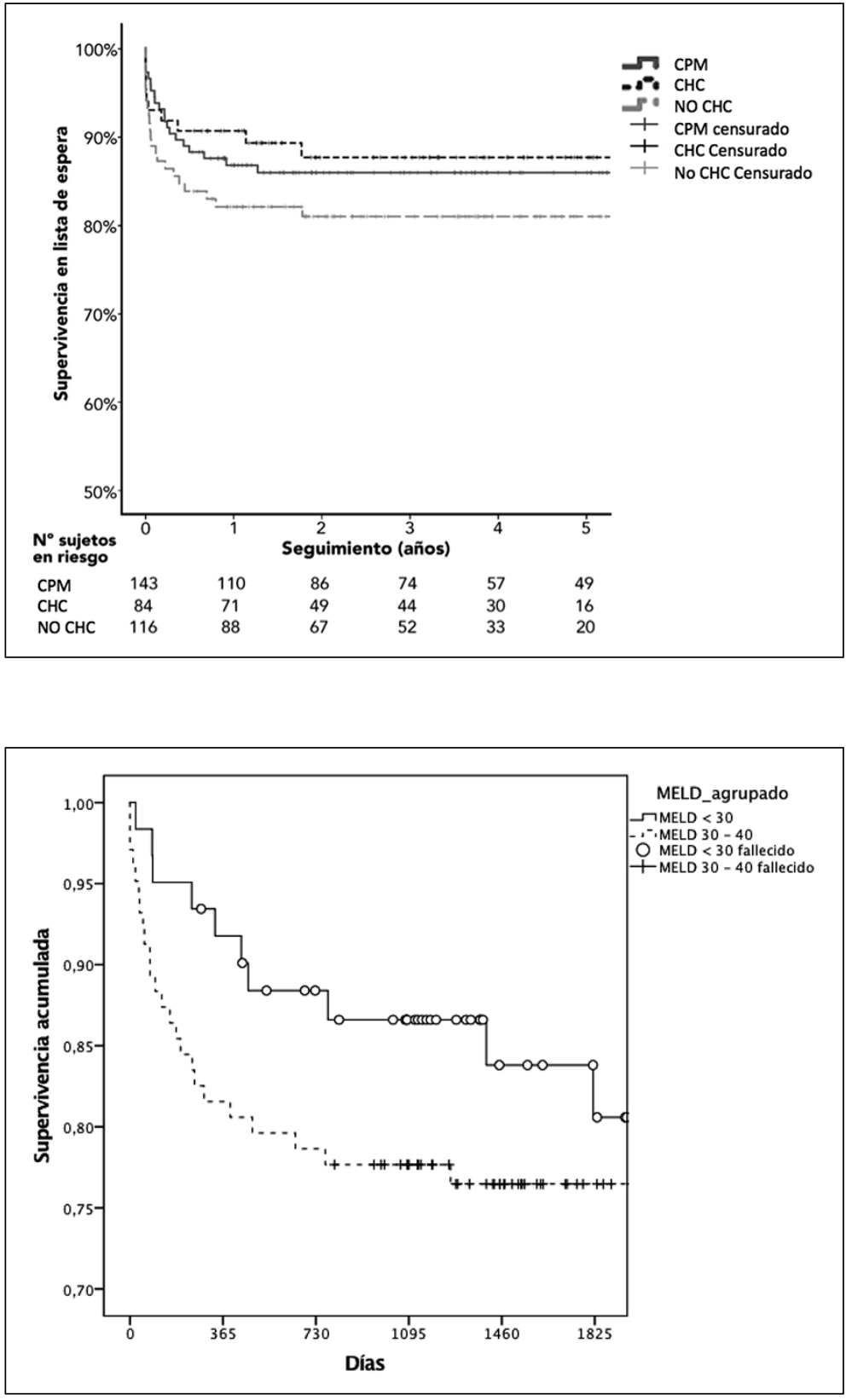

Figura 5. Supervivencia post trasplante en pacientes cirróticos (CPM), con carcinoma hepatocelular (CHC) y excepciones no carcinoma hepatocelular (No $\mathrm{CHC}$ ).

Figura 6. Supervivencia postrasplante en pacientes cirróticos (grupo CPM) según MELD agrupado al momento del trasplante.

de pacientes con cirrosis en Chile, que comparó el sistema de Child- Pugh con el sistema MELD, también se encontró una mejor correlación con este último sistema con mortalidad a un año ${ }^{20}$.

En el presente estudio se detectaron una gran cantidad de pacientes que fueron alistados por excepciones al MELD-Na real, lo que distorsiona su uso. De los pacientes adultos alistados en forma electiva, solo un tercio se priorizaron de acuerdo a su MELD-Na real, un tercio fueron priorizados por tener hepatocarcinoma y otro tercio por otras excepciones. Estas excepciones generaron inequidad en el acceso al trasplante. Los pacientes alistados por MELD-Na real tuvieron una mayor 
probabilidad de fallecer en la espera, comparados con los pacientes alistados por CHC y otras excepciones no CHC. Sin embargo, el tiempo promedio de espera al trasplante fue mayor en la excepciones comparado con los alistados por MELD real. Los pacientes con $\mathrm{CHC}$ tuvieron mayor probabilidad de trasplantarse y hubo menos fallecimientos mientras permanecían en lista de espera, a pesar de permanecer por más tiempo en ellas.

Por otro lado, la supervivencia posterior al $\mathrm{TH}$ fue buena en todos los grupos evaluados, atribuible al perfeccionamiento de la técnica de trasplante, comparable con la de países con mayores tasas de donación y de trasplantes. Sin embargo, dentro del grupo CPM, al comparar la mortalidad postrasplante se encontró una menor supervivencia en aquellos con MELD-Na $\geq 30$ al momento del trasplante ( $81 \%$ vs $91 \%$ al año), la cual aún es aceptable.

Una limitación común a una revisión retrospectiva es la dificultad en obtener datos. No se logró obtener datos suficientes para llegar a un análisis adecuado para cada una de las causas de excepción, las que debieron ser analizadas en su conjunto, a pesar de tener puntajes y progresiones diferenciadas. Se encontraron una gran cantidad de pacientes alistados por encefalopatía hepática. Si bien en otros estudios se ha detectado una mayor mortalidad independiente del MELD en este grupo de pacientes ${ }^{21}$, la evaluación de la gravedad puede ser subjetiva. No hay evidencia de que el prurito refractario sea un mayor riesgo de mortalidad para pacientes con enfermedades colestásicas, aunque la calidad de vida puede verse muy deteriorada. El trasplante de hígado puede ofrecer mejor calidad de vida, sin embargo, no hay un consenso en la priorización sistemática para esta indicación. En el caso de la ascitis refractaria, se han establecido criterios bastante estrictos para la inclusión en la lista de espera (al menos 6 punciones evacuadoras en los últimos 6 meses). En este grupo, la mortalidad puede ser de $50 \%$ al año sin trasplante ${ }^{22}$.

La priorización para TH usando el sistema de puntaje MELD o MELD-Na, utilizando puntajes operacionales para situaciones de excepción no ha disminuido la tasa de salida de lista de espera por muerte o deterioro clínico en Chile, como si ha sido demostrada en otros países. Al igual que en Chile, en Estados Unidos de Norteamérica, después de la introducción de la puntuación MELD, el número absoluto de pacientes alistados disminuyó, y el acceso a TH aumentó ${ }^{18}$. La adopción de la puntuación MELD en Argentina en 2005 resultó en una mejor asignación de órganos hepáticos, debido a una disminución significativas en la muerte en lista de espera (29,5\% versus $22,7 \%)$, $\mathrm{y}$ tasas de salida de lista y en el tiempo de espera al TH (108 versus 47 días), a pesar de un menor acceso a $\mathrm{TH}(61,3 \% \text { versus } 42,6 \%)^{14}$.

En Chile, la tasa de donación no ha mejorado significativamente en los últimos 10 años, a pesar de los grandes esfuerzos, recursos y campañas. Esto podría explicar la gran proporción de pacientes que aún fallecen en lista de espera. La asignación de puntuación MELD operacional permite una priorización mayor a pacientes con MELD-Na bajo, pero con otras condiciones que dan mayor mortalidad, las que deben ajustarse cuidadosamente para evitar que algunos grupos de pacientes se vean perjudicados.

\section{Referencias}

1. Hepp J, Zapata R, Buckel E, Martínez J, Uribe M, Díaz JC, et al. [General considerations, indications and contraindications for liver transplantation in Chile: a multicenter consensus development document]. Rev Med Chile 2008; 136 (6): 793-804.

2. Díaz LA, Norero B, Lara B, Robles C, Elgueta S, Humeres $\mathrm{R}$, et al. Prioritization for liver transplantation using the MELD score in Chile: Inequities generated by MELD exceptions: A collaboration between the Chilean Liver Transplant Programs, the Public Health Institute and the National Transplant Coordinator. Ann Hepatol 2019; 18 (2): 325-30.

3. Contreras A, McCormack L, Andraus W. Current status of liver transplantation in Latin America. Int J Surg 2020. PMID: 32247089.

4. Dutkowski P, Oberkofler CE, Bechir M, Mullhaupt B, Geier A, Raptis DA, et al. The model for end-stage liver disease allocation system for liver transplantation saves lives, but increases morbidity and cost: a prospective outcome analysis. Liver Transpl 2011; 17 (6): 674-84.

5. Malinchoc M, Kamath PS, Gordon FD, Peine CJ, Rank J, ter Borg PC. A model to predict poor survival in patients undergoing transjugular intrahepatic portosystemic shunts. Hepatology 2000; 31 (4): 864-71.

6. Wiesner R, Edwards E, Freeman R, Harper A, Kim $\mathrm{R}$, Kamath $\mathrm{P}$, et al. Model for end-stage liver disease (MELD) and allocation of donor livers. Gastroenterology 2003; 124 (1): 91-6. 
7. Ruf AE, Kremers WK, Chavez LL, Descalzi VI, Podesta LG, Villamil FG. Addition of serum sodium into the MELD score predicts waiting list mortality better than MELD alone. Liver Transpl 2005; 11 (3): 336-43.

8. Kim WR, Biggins SW, Kremers WK, Wiesner RH, Kamath PS, Benson JT, et al. Hyponatremia and mortality among patients on the liver-transplant waiting list. $\mathrm{N}$ Engl J Med 2008; 359 (10): 1018-26.

9. Mazzaferro V, Regalia E, Doci R, Andreola S, Pulvirenti A, Bozzetti F, et al. Liver transplantation for the treatment of small hepatocellular carcinomas in patients with cirrhosis. N Engl J Med 1996; 334 (11): 693-9.

10. Swanson KL, Wiesner RH, Krowka MJ. Natural history of hepatopulmonary syndrome: Impact of liver transplantation. Hepatology 2005; 41 (5): 1122-9.

11. Swanson KL, Wiesner RH, Nyberg SL, Rosen CB, Krowka MJ. Survival in portopulmonary hypertension: Mayo Clinic experience categorized by treatment subgroups. Am J Transplant 2008; 8 (11): 2445-53.

12. DuBrock HM, Goldberg DS, Sussman NL, Bartolome SD, Kadry Z, Salgia RJ, et al. Predictors of Waitlist Mortality in Portopulmonary Hypertension. Transplantation 2017; 101 (7): 1609-15.

13. Stewart CA, Malinchoc M, Kim WR, Kamath PS. Hepatic encephalopathy as a predictor of survival in patients with end-stage liver disease. Liver Transpl 2007; 13 (10): 1366-71.

14. Cejas NG, Villamil FG, Lendoire JC, Tagliafichi V, Lopez A, Krogh DH, et al. Improved waiting-list outcomes in Argentina after the adoption of a model for end-stage liver disease-based liver allocation policy. Liver Transpl 2013; 19 (7): 711-20.

15. Hong G, Lee KW, Suh S, Yoo T, Kim H, Park MS, et al. The model for end-stage liver disease score-based system predicts short term mortality better than the current
Child-Turcotte-Pugh score-based allocation system during waiting for deceased liver transplantation. J Korean Med Sci 2013; 28 (8): 1207-12.

16. Francoz C, Belghiti J, Castaing D, Chazouilleres O, Duclos-Vallee JC, Duvoux C, et al. Model for end-stage liver disease exceptions in the context of the French model for end-stage liver disease score-based liver allocation system. Liver Transpl 2011; 17 (10): 1137-51.

17. Montano-Loza AJ, Duarte-Rojo A, Meza-Junco J, Baracos VE, Sawyer MB, Pang JX, et al. Inclusion of Sarcopenia Within MELD (MELD-Sarcopenia) and the Prediction of Mortality in Patients With Cirrhosis. Clin Transl Gastroenterol 2015; 6: e102.

18. Moylan CA, Brady CW, Johnson JL, Smith AD, Tuttle-Newhall JE, Muir AJ. Disparities in liver transplantation before and after introduction of the MELD score. JAMA 2008; 300 (20): 2371-8.

19. Cholongitas E, Marelli L, Kerry A, Goodier DW, Nair $\mathrm{D}$, Thomas $\mathrm{M}$, et al. Female liver transplant recipients with the same GFR as male recipients have lower MELD scores-a systematic bias. Am J Transplant 2007; 7 (3): 685-92.

20. Sanhueza E, ContrerasJ, Zapata R, Sanhueza M, Elgueta F, López C, et al. Evaluación comparativa entre MELD y Child-Pugh como escalas pronósticas de sobrevida en pacientes con cirrosis hepática en Chile. Rev Med Chile 2017; 145: 17-24.

21. Wong RJ, Gish RG, Ahmed A. Hepatic encephalopathy is associated with significantly increased mortality among patients awaiting liver transplantation. Liver Transpl 2014; 20: 1454-61.

22. Moreau R, Delegue P, Pessione F, Hillaire S, Durand F, Lebrec D, Valla DC. Clinical characteristics and outcome of patients with cirrhosis and refractory ascites. Liver Int 2004; 24: 457-64. 